\title{
Silicon core fibers for integrated nonlinear systems
}

\author{
Anna C. Peacock \\ Optoelectronics Research Centre, University of Southampton \\ Southampton SO17 1BJ, United Kingdom \\ acp@orc.soton.ac.uk
}

\begin{abstract}
This paper will review progress in the development of nonlinear devices from the silicon core fibre platform, with a focus on their integration with existing fibre infrastructures. The nonlinear performance will be benchmarked through demonstrations of all-optical wavelength conversion and supercontinuum generation.
\end{abstract}

Index Terms-Nonlinear Fiber Optics; Fiber Fabrication; Silicon Photonics

\section{INTRODUCTION}

Silicon core fibers (SCFs) represent an emerging platform that combines the benefits of the fiber geometry with the advantages of semiconductor material systems [1]. As these fibers are clad in silica, they are robust, stable, and fully compatible with standard fibre fabrication procedures, thus increasing the device yield and reducing costs [2]. Moreover, these fibers are compatible with traditional post-processing techniques such as tapering, which can be used to precisely control the waveguide parameters, useful for phase-matching nonlinear processes, and improving coupling to other fiber components [3]. In this paper, I will review our efforts regarding the nonlinear characterization and application of SCFs over an extended wavelength range, covering the telecommunications band up to the mid-infrared. Our work in this area has shown that this new fiber technology has great potential for the development of a wide range of all-fiber integrated nonlinear systems, including wavelength convertors, amplifiers and broadband sources.

\section{FIBER FABRICATION}

The SCFs used in our work are fabricated via the molten core drawing (MCD) method [2]. In this approach, the silicon material is packed inside a glass tube to create a millimeter sized preform, which is then heated and drawn down into a fiber with micrometer dimensions. Owing to the high temperatures used in the drawing process, this method is currently restricted to producing SCFs with polycrystalline core materials, with core diameters on the order of several micrometers or larger. Thus, in order to reduce the core sizes down to the few-micrometer diameters needed to achieve efficient nonlinear processing, we make use of a post-draw tapering procedure. This procedure offers the added advantage in that by melting and recrystallizing the silicon core in a controlled fashion, it is possible to improve the material quality and thus reduce the optical losses to values comparable to their single-crystal silicon waveguide counterparts [4].
Fig. 1 displays a microscope image of a tapered SCF, illustrating the ability to control the core and cladding dimensions along the length. The inset shows a cross-section of a SCF fabricated via the MCD method, highlighting the welldefined high index core material with a diameter of $\sim 10 \mu \mathrm{m}$. For reference, typical loss values for the as-drawn fibers are around $10-15 \mathrm{~dB} / \mathrm{cm}$, and these can be reduced to around $1-2 \mathrm{~dB} / \mathrm{cm}$ after tapering [5].

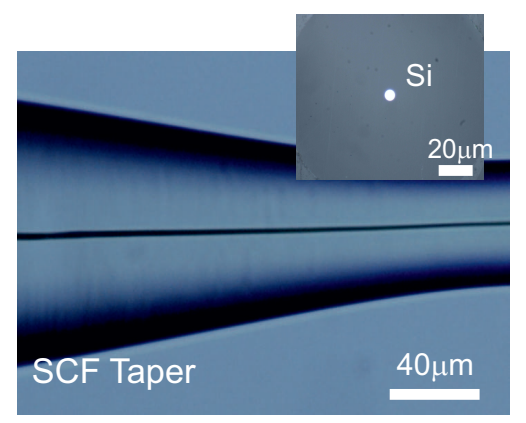

Fig. 1. Tapered silicon core fiber; inset shows the fiber cross-section.

\section{RESUlTS AND DiscUSSION}

\section{A. Nonlinear Characterization}

Although these loss values are still high for an optical fiber, owing to the large nonlinear coefficients of the silcon core materials they are nevertheless sufficient to allow for the first demonstrations of nonlinear propagation and characterization of this fiber class. To illustrate this, Fig. 2 shows examples of (a) all-optical wavelength conversion and (b) broadband supercontinuum generation obtained in the tapered SCF platform. Fig. 2(a) shows the results of four-wave mixing (FWM) wavelength conversion of optical signals in the telecoms band, obtained using a low power $(\sim 0.63 \mathrm{~mW}$ average, $\sim 17 \mathrm{~W}$ peak) femtosecond pump pulse source [6]. Broadband conversion can be observed for different signals $(1560-1670 \mathrm{~nm})$, which is only limited by the tuning range of our CW laser source. The signal gain peaks at $\sim 1570 \mathrm{~nm}$ with a value of $\sim 9 \mathrm{~dB}$, which is the highest parametric gain reported in a crystalline silicon waveguide in the telecoms band to date. Fig. 2(b) shows broadband, almost two-octave spanning $(1.6-3.5 \mu \mathrm{m})$, supercontinuum generation obtained in a tapered SCF, optimised for transmission in the midinfrared region [5]. Specifically, the fiber was designed to have a large tapered output coupler, to minimise interaction 


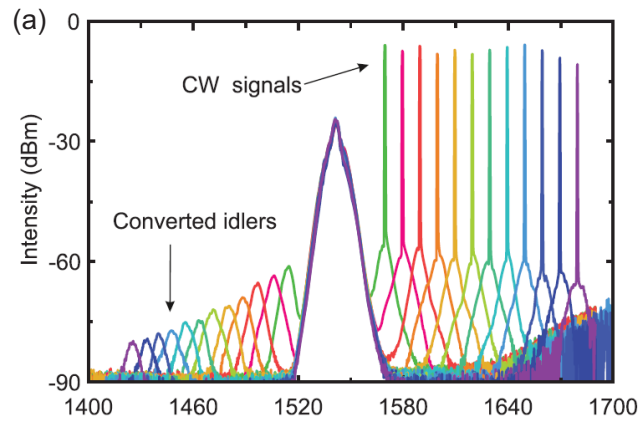

(b)

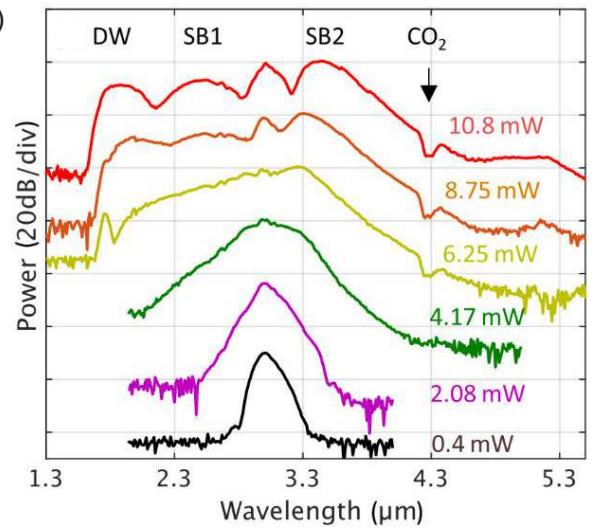

Fig. 2. (a) Overlapping output spectra showing FWM wavelength conversion of various telecom signals in a tapered SCF. (b) Supercontinuum spectra generated in a tapered SCF when pumped in the mid-infrared region.

of the generated mid-infrared light with the lossy cladding. When pumped with a $3 \mu \mathrm{m}$ pulse source, chosen to minimize the effects of two-photon absorption, we were able to achieve a power conversion efficiency of $60 \%$, which is higher than previous demonstrations in group IV-based waveguides. As a result, we were able to achieve an average supercontinuum power of $\sim 6 \mathrm{~mW}$ at the output, which is sufficient for many practical applications.

\section{B. SCF integration}

Despite their physical similarity to single mode fibers (SMFs), coupling light efficiently into and out of the SCFs remains a difficult task due to the high refractive index of silicon, which results in significant reflection losses and a large mode mismatch. We have recently developed a modified tapering procedure that enables us to produce nano-spike couplers on the ends of the SCFs, similar to those employed by the planar community [3]. These spikes can be cleaved and spliced to standard SMF using a modified fusion splicing technique to obtain a robust joint, as shown in Fig. 3. Optical transmission measurements of the integrated SCF-SMF device have revealed that the coupling loss due to the nano-spike is around $\sim 3-4 \mathrm{~dB}$, which is significantly lower than our freespace coupling approach, and numerical simulations indicate that this could be reduced to $\sim 0.5 \mathrm{~dB}$.

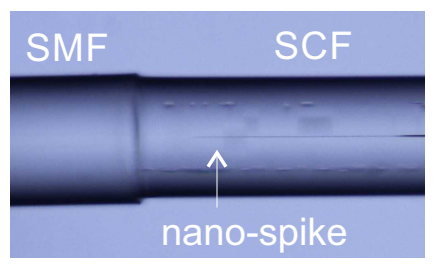

Fig. 3. Spliced SCF to SMF, facilitated via a silicon nano-spike coupler.

\section{CONCLUSION}

Silicon core fibres have been fabricated and characterized for use in nonlinear applications across a broad wavelength region, with device performances that are comparable, or better, than their planar counterparts. It has been shown that the SCFs can be directly integrated with conventional glass fibers, opening a route for robust and efficient all-fiber nonlinear systems.

\section{ACKNOWLEDGMENT}

This work was supported by EPSRC.

\section{REFERENCES}

[1] A. C. Peacock, U. J. Gibson, and J. Ballato, "Silicon optical fibresPast, present, and future," Adv. Phys.: X 1, 114-127 (2016).

[2] J. Ballato, T. Hawkins, P. Foy, R. Stolen, B. Kokuoz, M. Ellison, C. McMillen, J. Reppert, A. M. Rao, M. Daw, S. Sharma, R. Shori, O. Stafsudd, R. Rice, and D. R. Powers, "Silicon optical fiber," Opt. Express 16, 18675-18683 (2008).

[3] H. Ren, O. Aktas, Y. Franz, A. F. J. Runge, T. Hawkins, J. Ballato, U. J. Gibson, and A. C. Peacock, "Tapered silicon core fibers with nanospikes for optical coupling via spliced silica fibers," Opt. Express 25, 24157-24163 (2017).

[4] F. H. Suhailin, L. Shen, N. Healy, L. Xiao, M. Jones, T. Hawkins, J. Ballato, U. J. Gibson, and A. C. Peacock, "Tapered polysilicon core fibers for nonlinear photonics," Opt. Lett. 41, 1360-1363 (2016).

[5] H. Ren, L. Shen, A. F. J. Runge, T. Hawkins, J. Ballato, U. J. Gibson, and A. C. Peacock, "Low-loss silicon core fibre platform for mid-infrared nonlinear photonics," Light: Sci. \& Appl. 8, 105 (2019).

[6] D. Wu, L. Shen, H. Ren, J. Campling, T. W. Hawkins, J. Ballato, U. J. Gibson, and A. C. Peacock, "Net optical parametric gain in a submicron silicon core fiber pumped in the telecom band," APL Photonics 4, 086102 (2019). 